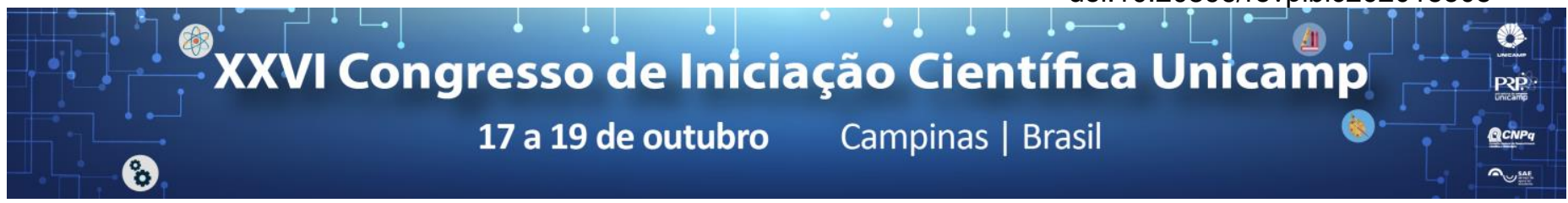

\title{
GELLAN AEROGEL PRODUCTION APPLYING SUPERCRITICAL TECHNOLOGY
}

\section{Gabriela F. Freitas*, Juliane Viganó, Julian Martínez.}

\begin{abstract}
Aerogels are materials with an open porous, low density and high surface area, which make them an interesting material to carry target compounds. The proposal of this work was to produce gellan aerogels and to study the effect of the diffusion method and internal setting method, the temperature of the gellan pre-treatment, and the addition of inulin on the aerogel shrinkage during the process. The aerogels were produced through hydrogel formulation, solvent exchange to obtain alcogel, and supercritical drying with carbon dioxide. The addition of inulin has reduced the shrinkage, but gelling methods to avoid the inulin loss must be studied, as the oil containing the gelling agent.
\end{abstract}

\section{Key words:}

Aerogel, Gellan, Supercritical Technology.

\section{Introduction}

Aerogel is defined as an open porous and light material, which has the capacity of loading target compounds and providing controlled release. Due to those characteristics, the polysaccharide-based biodegradable aerogels are studied to be used in food and drugs. A promising polysaccharide is gellan gum, which produces a strong gel in the presence of calcium. Based on that, the objective of this work was to produce gellan aerogels with low shrinkage percentage, obtained through supercritical drying with $\mathrm{CO}_{2}$.

\section{Results and Discussion}

Polysaccharide solutions were prepared with gellan $(0.5$, 0.75 e $1 \% \mathrm{w} / \mathrm{w})$. Each polysaccharide solution was dripped through a nozzle $(0.58 \mathrm{~mm})$ into a crosslinking solution containing different ionic strength calcium chloride $(0.03,0.06$ e $0.09 \mathrm{M})$. Fig 1 shows the shrinkage results. The highest shrinkage percentages were achieved after supercritical drying. The gellan particles 0.75 and $1 \%$ show smaller global shrinks.

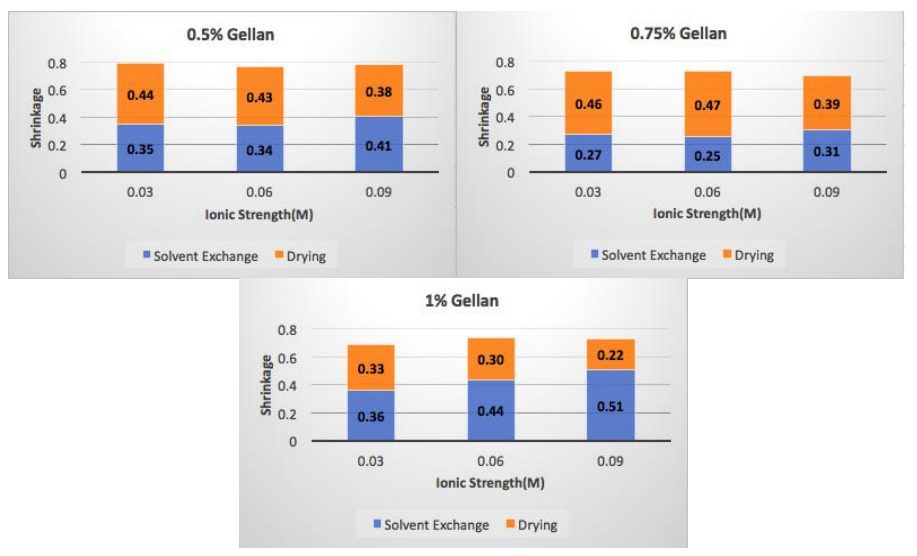

Fig 1.Effect of gellan concentration and the crosslinking ionic strength on the shrinkage during the process.

The effect of gellan treatment temperature (room temperature, 50, 70 e $90^{\circ} \mathrm{C}$ ), and the addition of inulin $(10 \% \mathrm{w} / \mathrm{w})$ to the gellan $0.75 \%$ were tested. The gels were produced using membrane (14 kDa) to obtain monoliths. The addition of inulin has shown more resistance to shrinkage during the process. The results are shown in Fig 2 and 3.

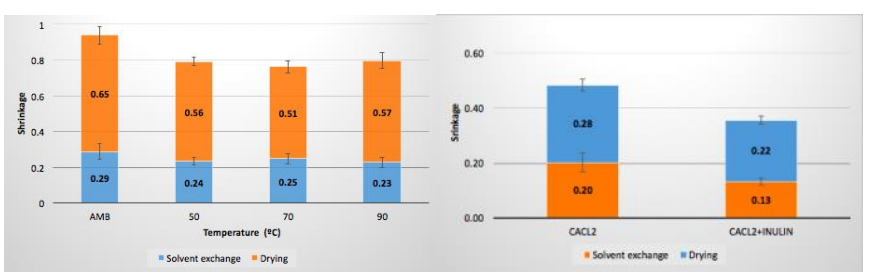

Fig 2. Shrinkage depending on the Temperature.

Fig 3.Effect of inulin on shrinkage.

The internal gelling using glacial acetic acid $(0.5,1 \mathrm{e}$ $1.5 \% \mathrm{w} / \mathrm{w})$ and soy oil containing $1 \% \mathrm{w} / \mathrm{w}$ Span 80 , varying the $\mathrm{CaCO}_{3}$ concentration $(0.05,0.1,0.15$ and 0.2 $\mathrm{g} / \mathrm{g}$ gellan) was studied. There was no difference in the shrinkage, thus using smaller reagent concentrations on the product formulation is more viable.

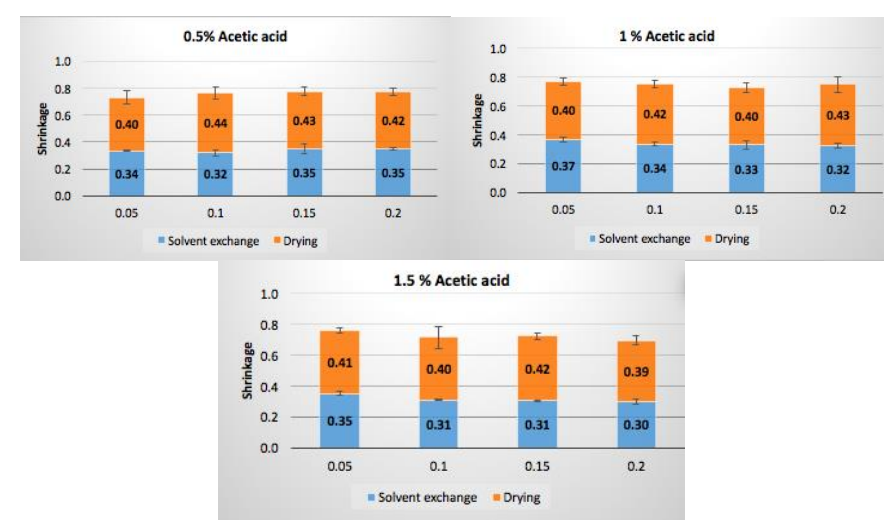

Fig 4. Effect of the internal setting method on the shrinkage.

\section{Conclusions}

Raising the gellan concentration decreased the shrinkage. The gel prepared with pre-treated gellan at 70 ${ }^{\circ} \mathrm{C}$ showed lower shrinkage percentage. The internal setting method is advantageous to avoid loss of inulin to the crosslinker. Therefore, the application of inulin in aerogels produced by internal setting method is suggested. 